\title{
Novel Twig Sampling Method by Unmanned Aerial Vehicle (UAV)
}

\begin{abstract}
Florian Käslin ${ }^{1}$, Thomas Baur ${ }^{1}$, Philip Meier ${ }^{1 \dagger}$, Patrick Koller ${ }^{1,2}$, Nina Buchmann ${ }^{1 \dagger}$, Petra D'Odorico ${ }^{1,3}$ and Werner Eugster ${ }^{1 * \dagger}$
\end{abstract}

${ }^{1}$ Department of Environmental Systems Science, Institute of Agricultural Sciences, ETH Zurich, Zurich, Switzerland, ${ }^{2}$ Federal Office of Meteorology and Climatology MeteoSwiss, Zurich, Switzerland, ${ }^{3}$ Department of Biology, University of Toronto at

Mississauga, Mississauga, ON, Canada

\section{OPEN ACCESS}

Edited by:

Jeffrey M. Warren,

Oak Ridge National Laboratory (DOE),

United States

Reviewed by:

Teresa E. Gimeno,

Basque Centre for Climate Change,

Spain

Misha Krassovski,

Oak Ridge National Laboratory (DOE),

United States

*Correspondence:

Werner Eugster

werner.eugster@usys.ethz.ch orcid.org/0000-0001-6067-0741

${ }^{\dagger}$ Philip Meier orcid.org/0000-0001-7789-7002 Nina Buchmann orcid.org/0000-0003-0826-2980

Specialty section

This article was submitted to

Forest Ecophysiology,

a section of the journal

Frontiers in Forests and Global

Change

Received: 16 July 2018 Accepted: 05 September 2018 Published: 18 October 2018

Citation: Käslin F, Baur T, Meier P, Koller P, Buchmann N, D'Odorico P and Eugster W (2018) Novel Twig Sampling Method by Unmanned Aerial Vehicle (UAV) Front. For. Glob. Change 1:2 doi: 10.3389/ffgc.2018.00002
Biophysical and biochemical traits of foliage and twigs at the top of tree canopies provide essential information on trees' ecophysiology. Conventional methods used for canopy sampling are typically time consuming and costly, while the very canopy tops are still out of reach unless a canopy crane is used. Thus, we developed a novel twig sampling method using a device attached to a drone that allows to grasp and cut small twigs and bring them to the ground for immediate analysis. This "Flying Tree Top Sampler" (FITS) complements existing methods used in tree canopy research. This paper describes the method to make it openly available to interested users, provides the necessary technical details, and reports on a proof-of-concept application by probing a single tree during senescence. In this demonstration example we show that the ability to investigate the top part of the tree leads to the clear finding that chlorophyll content of the top leaves is significantly lower than that of leaves in the lower canopy. Without the samples collected by the FITS, the decrease of chlorophyll content with height would not have been of statistical significance using a $p=0.05$ significance threshold. Besides its advantages, the existing limitations of the FTTS are discussed, and suggestions for future developments are provided.

Keywords: tree canopy sampling, leaf sample collection, ground truthing, leaf physiological measurements, leaf chlorophyll, tree senescence, unmanned aerial vehicle

\section{INTRODUCTION}

Forest canopies play an important role globally in terms of biodiversity, biospheric-atmospheric gas exchange, and ecosystem services (Brockerhoff et al., 2017). However, canopy science has been hindered in most ecosystems by a simple challenge (e.g., Parker et al., 1992; Stork et al., 1997; Hallé et al., 2000; Barker and Pinard, 2001): How to reach the top? Here we report on a novel, cost efficient device attached to a drone, the Flying Tree Top Sampler (FTTS). It grasps and cuts twigs (and small branches) from the very canopy top and safely carries them back to the operators. Researchers studying the ecophysiology of trees and forest canopies require fresh and intact foliage samples from a large number of trees sampled within a short time span to analyze, for example, chlorophyll or fluorescence, determine light response curves of photosynthesis, or measure stomatal conductance. Sampling and measurements have to be carried out quickly, otherwise rapid changes in tissue water status may trigger unwanted changes in biophysical and 
biochemical traits of leaves and twigs (Larcher, 2003), such as stomatal aperture, which adjusts to environmental conditions within minutes (Chapin et al., 2002).

As trees and canopies are very diverse in size and shape, a wide range of methods for sampling has been employed in canopy science to date (Sweney and Jones, 1974; Sutton, 2001; Nakamura et al., 2017). Smaller trees have been sampled with ladders, extendable tree pruners, or hydraulic forklifts, to name just a few. These methods have disadvantages in both applicability to tall tree canopies, tree accessibility and difficult terrain, in particular for reaching top canopy twigs of trees within dense stands. Other studies worked with shotguns and crossbows to obtain foliage from upper canopy twigs (Sweney and Jones, 1974; Lowman and Moffett, 1993). However, when twigs cannot be seen directly, it is likely that the sample obtained does not only contain foliage from the upper canopy, and thus may not be representative of the tree top. For tall trees and top canopy sampling, simple methods are very limited, thus canopy rafts (Hallé et al., 2000), walkways (Muul and Liat, 1970; Sutton, 2001) and cranes (Parker et al., 1992; Stork et al., 1997) have been used. Due to the high initial costs to install any of these types of infrastructure, tree climbers are still the most common way to sample tall tree canopies. Tree climbers require almost no initial investment, but are expensive to sample the tops of tall trees over larger areas. Moreover, foliage visible to airborne sensors is typically at the very top of tree canopies and is thus often out of (safe) reach, even for the best of tree climbers. Hence, we developed and tested the deployment of a FTTS to reach the otherwise unreachable very top of tall tree canopies. This paper describes the method to make it openly available to interested users, provides the necessary technical details, and reports on a proof-of-concept application by probing a single tree during senescence.

\section{MATERIALS AND METHODS}

\subsection{Flying Tree Top Sampler (FTTS)}

The device (Figure 1A) consists of a twig sampling mechanism (with the grasping, cutting and carrying mechanisms) attached to a multicopter drone by a tether. Two people are required to operate the device: one person flies the drone within line of sight, another one operates the twig sampling mechanism. Harvesting one twig sample from the top of a tree canopy takes less than five minutes once the FTTS is ready for take-off. The twig sampling mechanism weighs $870 \mathrm{~g}$, the tether weighs 560 g. Thus, many drones are capable of carrying our prototype twig sampling mechanism.

\subsection{Twig Sampling Mechanism}

All components that are not commercially available (grippers, housing, etc.) were custom-made with a 3D printer (Maker's Tool Works, MendelMax 3 Full Kit, Desert Hot Springs, CA, USA). A circular saw (WESA, Type C, HSS, $50 \mathrm{~mm}$ diameter, $1 \mathrm{~mm}$ thickness, Haryana, India) is used for twig cutting. Three pairs of grippers driven by a single servo motor (TrackStar, TS-621MG, Digital 1/8 Scale Truggy Steering Servo 25T, Hobbyking.com, Hong Kong, China; torque $2.12 \mathrm{kNm}$, reaction time $0.14 \mathrm{~s}$, weight $57 \mathrm{~g}$ ) are used to position the saw perpendicular to a target twig and to grasp the twig. The two lower pairs of grippers (Figure 1D) exert their force in the plane of the circular saw. The third and uppermost pair of grippers is positioned somewhat above (Figure 1B) to align the twig sampling mechanism toward the twig and absorb the angular momentum of the rotating saw.
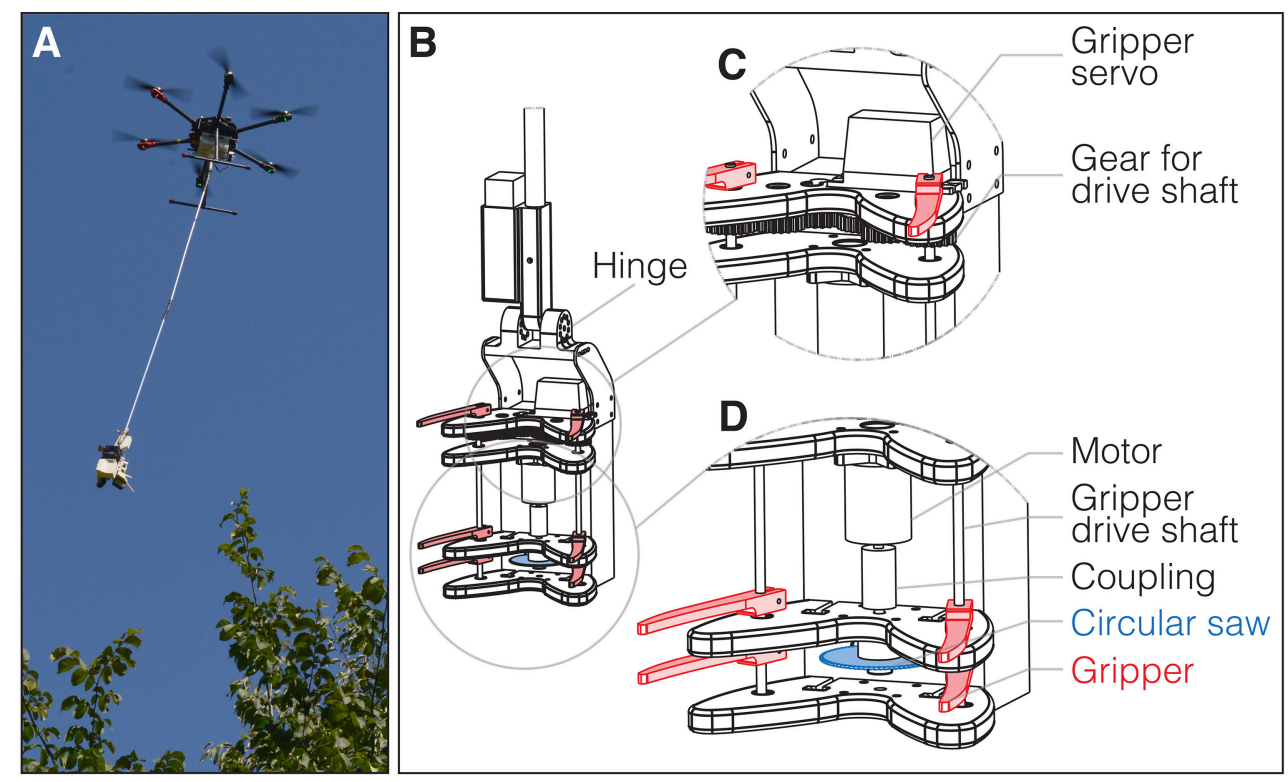

FIGURE 1 | The Flying Tree Top Sampler (FITS). (A) The full system consisting of a drone with a twig sampling mechanism suspended below. (B) The twig sampling mechanism with details showing the grippers to grasp and hold a twig (C,D), and the circular saw (D) to cut it off the tree. 
Additionally, it ensures that the twig is held in vertical position once it is cut and thus prevents the sample from being lost. The speed of the servo transmission is optimized to provide the most reliable cutting action. The saw blade is driven by a brushless motor (DYS, BE2217-7, 1200KV, Kawasaki, Japan) and rotates at more than 10,000 rotations per minute. The sensing unit consists of an ultrasonic proximity sensor (ITeadStudio, HC-SR04, 2$500 \mathrm{~cm}, 0.3 \mathrm{~mm}$ resolution, Shenzhen, China) that is used to detect a twig within reach of the grippers. The proximity sensor is connected to an Arduino Nano microcontroller (Gravitech LLC, Minden, NV, USA). A wireless interface consisting of a remote control/transmitter (Turnigy, RF9X V2, 2.4 GHz, Hong Kong, China) and a receiver (Turnigy, TGY-iA10, $2.4 \mathrm{GHz}, 10$ channel, Hong Kong, China) is used to connect to the operator's console from which the twig sampling mechanism is enabled to grasp, cut and hold the twig automatically. However, all of these actions can be overridden by the operator via remote control. The system is powered by a battery pack allowing for 30 minutes of operation of both flight and twig sampling operations.

\subsection{Tether and Drone}

The twig sampling mechanism is suspended below a multicopter drone (Tarot X6 Pro with 6 rotors, Wenzhou, China) with a tether consisting of three one-meter long sections of aluminum tubing ( $16 \mathrm{~mm}$ outer and $14.3 \mathrm{~mm}$ inner diameter) with custommade motion dampers (i.e., heat shrink tubing filled with sand) between each section. These tubes dampen the pendulum effect of the twig sampling mechanism dangling below the drone, stabilizing the twig sampling mechanism's motion when the drone is transitioning from flight to hovering. At the distal end of the tether, the twig sampling mechanism is attached with a hinge that allows for a passive $\pm 90^{\circ}$ adjustment of the saw (Figure 1B) when the grippers grasp a target twig. The drone is controlled by a remote control (Turnigy, TGY-i10, $2.4 \mathrm{GHz}, 10$ channel, Hong Kong, China) equipped with a First Person View (FPV) system (camera: Aomway, $1200 \mathrm{TVL}$; lens: $2.8 \mathrm{~mm}$; field of view: $120^{\circ}$, charge-coupled device (CCD) sensor: 1/3" Sony HD, 22 g, Shenzen, China; transmitter: Fatshark, FSV2462, Shenzen, China; receiver: Boscam, FP632, $5.8 \mathrm{GHz}, 40$ channel, Shenzen, China) to ease the flying and sampling process for the two operators (see Section 2.5).

\subsection{Safety Precautions}

The circular saw is shrouded in a protective housing, with the grippers on both sides, leaving only a $40^{\circ}$ arc of the rotating saw blade exposed to the twig when the grippers are wide open, thereby preventing unwanted objects from contacting the blade. User safety was a key requirement for the design, with a safety interlock protocol controlling the saw. The twig sampling mechanism is only activated when the operator has unlocked the system and a target twig is within $100 \mathrm{~cm}$ distance of the ultrasonic sensor. At any time, the operator can deactivate the twig sampling mechanism. This immediately turns off the saw motor and opens the grippers. A second safety feature is the quick release mechanism of the tether from the drone. Should any difficulties be experienced during flight, e.g., the twig sampling mechanism getting trapped in a tree, the flight operator can release the tether and safely fly the drone back to base. This feature can however also be used to safely land the drone (see Supplementary Material 1).

Although a drone without payload may be flown even in a fresh breeze (i.e., 9-11 $\mathrm{m} \mathrm{s}^{-1}$ ), the upper limit of secure operation of the FTTS, with its cutting device attached in the way presented in this paper, is limited to conditions with wind speeds not exceeding $4-5 \mathrm{~m} \mathrm{~s}^{-1}$.

\subsection{Flight Operation}

The drone with the attached twig sampling mechanism is controlled by two operators, one controlling the FTTS flight, the other one operating the twig sampling mechanism. Each operator has an independent remote control for his or her task: flying and sampling, respectively. They use a FPV live video feed from the drone which provides a camera view plus the status of various operating variables, such as height above ground level, global positioning system (GPS) location, position relative to the drone's take-off location (base station), radio signal strength, and battery

TABLE 1 | Data from Norway maple (Acer platanoides L.) measured on 12 October 2016 at the $\mathrm{CH}-\mathrm{CHA}$ Swiss FluxNet site, Switzerland.

\begin{tabular}{|c|c|c|c|c|c|}
\hline Exposure & Height (m) & Layer & Gain CCM & CFR & Chlorophyll $\left(\mathrm{mg} \mathrm{m}^{-2}\right)$ \\
\hline$N$ & 2 & Reference & 5 & 1.51 & 566 \\
\hline $\mathrm{N}$ & 2 & Reference & 4 & 1.63 & 642 \\
\hline $\mathrm{N}$ & 2 & Reference & 4 & 1.47 & 541 \\
\hline$N$ & 4 & Reference & 4 & 1.03 & 262 \\
\hline$N$ & 4 & Reference & 4 & 1.33 & 452 \\
\hline $\mathrm{N}$ & 4 & Reference & 4 & 1.41 & 503 \\
\hline $\mathrm{N}$ & 6 & Reference & 4 & 1.54 & 585 \\
\hline $\mathrm{N}$ & 6 & Reference & 4 & 1.45 & 528 \\
\hline$N$ & 6 & Reference & 4 & 1.35 & 465 \\
\hline$N$ & 8 & MidCanopy & 4 & 1.15 & 338 \\
\hline $\mathrm{N}$ & 8 & MidCanopy & 4 & 0.78 & 103 \\
\hline $\mathrm{N}$ & 8 & MidCanopy & 4 & 1.30 & 433 \\
\hline$N$ & 10 & CanopyTop & 4 & 0.80 & 116 \\
\hline $\mathrm{N}$ & 10 & CanopyTop & 4 & 0.97 & 224 \\
\hline $\mathrm{N}$ & 10 & CanopyTop & 4 & 0.92 & 192 \\
\hline S & 2 & Reference & 4 & 1.23 & 389 \\
\hline S & 2 & Reference & 4 & 1.43 & 515 \\
\hline S & 2 & Reference & 4 & 1.38 & 484 \\
\hline S & 4 & Reference & 4 & 1.24 & 395 \\
\hline S & 4 & Reference & 4 & 1.27 & 414 \\
\hline S & 4 & Reference & 4 & 1.34 & 458 \\
\hline S & 6 & Reference & 4 & 1.21 & 376 \\
\hline S & 6 & Reference & 4 & 1.15 & 338 \\
\hline S & 6 & Reference & 4 & 0.94 & 205 \\
\hline S & 8 & MidCanopy & 4 & 1.18 & 357 \\
\hline S & 8 & MidCanopy & 4 & 1.25 & 401 \\
\hline S & 8 & MidCanopy & 4 & 1.19 & 363 \\
\hline S & 10 & CanopyTop & 4 & 0.99 & 236 \\
\hline S & 10 & CanopyTop & 4 & 1.13 & 325 \\
\hline S & 10 & CanopyTop & 4 & 1.24 & 395 \\
\hline
\end{tabular}


voltage. The onboard flight control system of the FTTS (Tarot, ZYX-M, GPS, $116 \mathrm{~g}$, Wenzhou, China) is responsible for the technical flying.

\subsection{Chlorophyll Content Measurements}

Chlorophyll content of fresh leaves collected from one single tree (Table 1) was measured with a chlorophyll content meter (CCM-300, Opti-Sciences, Hudson, NH, USA) within one minute after the twig was cut. The gain of the CCM-300 (Table 1) indicates the integration time: with a gain setting of 4 the signal is averaged for five seconds; with a gain setting of 5 , the signal is averaged for 10 seconds. The CFR (Table 1) is the ratio of fluorescence emission at $735 \mathrm{~nm}$ normalized by the fluorescence emission at $700 \mathrm{~nm}$ (not used in this study). Three replications from each canopy height $(2,4,6,8$, and $10 \mathrm{~m})$ and tree side (north, south) were analyzed.

\subsection{Statistical Analyses}

Statistical analyses were done using R version 3.4.1 (R Core Team, 2016). Linear mixed effect models were calculated using the lme4 package using the restricted maximum likelihood (REML) approach, and estimated marginal means (EMMs) of linear mixed-effect models were calculated using the emmeans package (Searle et al., 1980; Lenth, 2018).

\section{PROOF-OF-CONCEPT APPLICATION OF THE FTTS}

\subsection{Case Study}

Applicability of the FTTS was successfully tested on broadleaf as well as conifer trees (see Supplementary Material 1). Here we present a case study in which we sampled a tree growing in an allée (Norway maple, Acer platanoides L.) near the $\mathrm{CH}-\mathrm{CHA}$ Swiss FluxNet site, Switzerland $\left(47^{\circ} 12^{\prime} 36.8^{\prime \prime} \mathrm{N}, 8^{\circ} 24^{\prime} 37.6^{\prime \prime} \mathrm{E}\right.$, $393 \mathrm{~m}$ a.s.l.) in October 2016 during early senescence and determined leaf chlorophyll contents from a subsample of leaves from each twig collected (Figure 2). Mean chlorophyll contents were not significantly different between the north side (shaded) and the south side (sunlit) of the tree at the highest level that could be reached without the FTTS, i.e., at $8 \mathrm{~m}$ above ground (Welch two-sample two-tailed $t$-test, $t=0.8313, d f=2.0789, p$ $=0.49$ ). However, the top canopy leaves at $10 \mathrm{~m}$ above ground that could only be collected via our novel FTTS revealed that the chlorophyll content in the south-exposed tree top was on average $80 \%$ larger than that in the corresponding north-exposed tree top (Welch two-sample one-tailed $t$-test, $p=0.036$ ). Contrastingly, the difference between chlorophyll contents on the north and south side of the entire tree was not significant ( $\mathrm{z}$ ratio $=-0.467$, $p=0.64$, adjusted for multiple testing using Tukey's approach). But a significant decrease in chlorophyll content with height was found (Figures 2B, Table 2). The canopy top samples collected with the FTTS had a significantly lower chlorophyll content

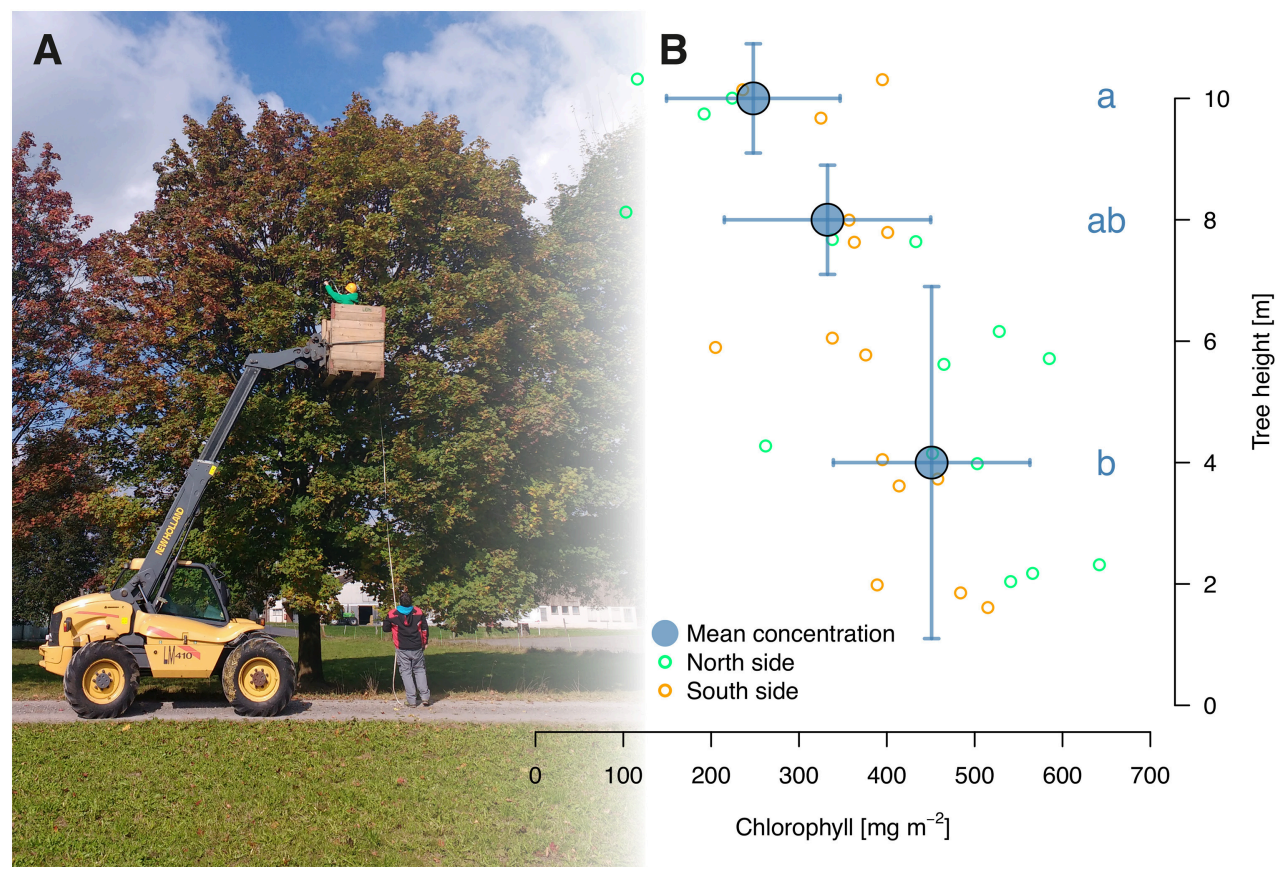

FIGURE 2 | Leaf chlorophyll content of a tree growing in an alleé during senescence. (A) Conventional fork-lift sampling at 2, 4, 6, and $8 \mathrm{~m}$ height. (B) Chlorophyll content on the sun exposed (south, orange circles) and shaded (north, green circles) side of the tree. The top canopy samples (10 m) were collected with a drone. Group means (filled circles) show main canopy ( $\leq 6 \mathrm{~m})$, upper canopy reachable by fork lift ( $8 \mathrm{~m})$, and top canopy sampled by the FTTS (10 m). Horizontal whiskers show the standard deviation from the mean, and vertical whiskers show the height range covered by the mean and standard deviation. Layers (a, ab, b) which have no letter in common are significantly different from each other $(p<0.05)$. See also Tables $\mathbf{2}-\mathbf{4}$. 
TABLE 2 | Analysis of variance (ANOVA) table of group means of chlorophyll contents shown in Figure 2, using three canopy heights (Reference $\leq 6 \mathrm{~m}$, MidCanopy $8 \mathrm{~m}$, CanopyTop $10 \mathrm{~m}$ ) and two exposures (N, S) obtained from linear mixed-effect model.

\begin{tabular}{lcccc}
\hline Source of variation & df & Sum squares & Mean squares & F ratio \\
\hline Height & 2 & 207,473 & 103,736 & 8.2314 \\
Exposure & 1 & 2,750 & 2,750 & 0.2182 \\
Total & 3 & 210,223 & & \\
\hline
\end{tabular}

TABLE 3 | Group means of measurement heights of the linear mixed-effect model (Table 2) obtained using the estimated marginal means (EMMs) approach.

\begin{tabular}{llllll}
\hline & & & & \multicolumn{2}{c}{ Confidence limits $^{\mathbf{c}}$} \\
\cline { 5 - 6 } Height & Mean $^{\mathbf{a}}$ & SE & $\mathbf{d f}^{\mathbf{b}}$ & Lower & Upper \\
\hline CanopyTop & 248.0 & 46.2 & $\infty$ & 157.4 & 338.6 \\
MidCanopy & 332.5 & 46.2 & $\infty$ & 241.9 & 423.1 \\
Reference & 451.0 & 27.1 & $\infty$ & 397.9 & 504.1
\end{tabular}

a Results are averaged over the levels of: Exposure

${ }^{b}$ Degrees-of-freedom method: asymptotic

c $95 \%$ confidence interval

TABLE 4 | Contrasts among measurement heights of the linear mixed-effect model (Table 2) obtained using the estimated marginal means (EMMs) approach.

\begin{tabular}{lccccc}
\hline Contrast & Estimate $^{\mathbf{a}}$ & SE & df & z ratio & p-value $^{\mathbf{b}}$ \\
\hline Reference-CanopyTop & 203.0 & 52.9 & $\infty$ & 3.836 & 0.0004 \\
Reference-MidCanopy & 118.5 & 52.9 & $\infty$ & 2.239 & 0.0648 \\
CanopyTop-MidCanopy & -84.5 & 64.8 & $\infty$ & -1.304 & 0.3931 \\
\hline
\end{tabular}

a Results are averaged over the levels of: Exposure.

${ }^{b} p$ value adjustment: Tukey method for comparing a family of 3 estimates.

$\left(248 \pm 46 \mathrm{mg} \mathrm{m}^{-2}\right.$, Table 3$)$ than the main canopy $(2 \pm 6 \mathrm{~m}$, $451 \pm 27 \mathrm{mg} \mathrm{m}^{-2}$, Table 3; $p<0.001$, Table 4). The highest layer accessible without the FTTS $(8 \mathrm{~m})$ had intermediate chlorophyll contents ( $333 \pm 46 \mathrm{mg} \mathrm{m}^{-2}$ ), but differed only marginally from the value observed in the main canopy ( $p=0.064$, Table 4$)$.

\subsection{Potential Applications of the FTTS}

In addition to being quick, this approach allows to access the very top of the canopy, which dominates overall tree photosynthesis (Niinemets, 2012). Moreover, it allows for sampling of extended areas and thus of a more representative part of a forest ecosystem than other methods. The FTTS further has the potential to be employed in challenging environments such as closed-canopy tropical forests, if an elevated platform is used for take-off and landing, and if the line-of-sight requirement for drone flying can be met. Calibration of remote sensing spectral proxies of photosynthesis acquired from drones, aircraft or satellites (Congalton and Green, 2009; Anderson and Gaston, 2013) will also greatly benefit from this novel sampling approach (Curran, 1989; Kokaly et al., 2009) which can be synchronized with the overflights (Jensen, 2007; Huete et al., 2014). Thus, the FTTS provides a fast, easy to use, cost efficient method to fulfill all these requirements.

\section{LIMITATIONS OF THE FTTS AND FUTURE DEVELOPMENTS}

Besides its potential for complementing traditional sampling methods, the FTTS also has its limitations. Although FPV technically enables the FTTS to fly anywhere, regulations in most countries still require that a drone is flown within line of sight of the pilot. Thus, in areas where the line-of-sight criterion is difficult to meet, also the applicability of an FTTS remains limited. This may be the case in dense forests in flat landscapes, hence we stated that an elevated platform may be needed for take-off and landing. Contrastingly, in mountainous regions like Switzerland, there is often an elevated topographical position where the pilot can have the necessary overview to fly a drone above a dense forest canopy.

Accurate geo-positioning may pose another limitation in narrow valleys or within tall buildings, where GPS positioning may be of low accuracy if there are not enough GPS satellites within view. Drone pilots tend to know these limitations, but scientists not familiar with GPS should be aware of the current limitations of geo-positioning of a drone.

The choice of a specific drone for an FTTS should also consider the fact that a higher payload could be used to carry a heavier battery to extend the operation period in a practical application. Using the prototype tested here with an operation time of $30 \mathrm{~min}$ it is possible to collect around six samples, e.g., from different trees, before the battery needs to be exchanged. Swapping the battery for a fully charged one typically takes one minute. A reduction in weight would also increase battery life and operation time of the FTTS. The prototype described here was specifically designed to prove the concept, and thus the weight of most components could be reduced in the future, namely the aluminum tubing used for the tether could be substituted by carbon fiber tubes.

Technical developments of drone autopilots will most likely lead to drones that are capable of flying collision-free within forest canopies in the near future. Once this skill is achieved, future developments of the FTTS should focus on benefiting from such possibilities, which will help to reduce the length of the suspension tether. Ultimately it may be possible to attach the cutting device rigidly to the body of the drone, which then could operate similar to a bird of prey, flying away with the cut twig from any angle it was cut. There will still be a limitation of the weight that the FTTS can carry away after cutting off the twig. With the $50 \mathrm{~mm}$ diameter saw used in our device the upper limit of twig diameter may be on the order of $20 \mathrm{~mm}$. But branches cut where the twig is of this diameter tend to be too heavy for the FTTS to carry it safely back to the operator.

\section{CONCLUSIONS}

Since remote sensing products from drones, aircraft, and satellites are more strongly influenced by top canopy leaves than by lower canopy leaves, ground truthing of tree canopies and forest stands will become more reliable when these top twigs become more easily accessible from trees spread over a larger surface area than what can be reached with a canopy crane, 
canopy rafts, or tree climbers. Our results suggest that using drones with a twig sampling mechanism such as the FTTS adds a new, complementary accession technique. This substantially expands our ability to sample the very tree tops, thus providing information about yet unexplored areas of tall forest canopies.

\section{AUTHOR CONTRIBUTIONS}

FK designed, produced and tested all technical components and wrote the first draft of the manuscript. WE initiated the project (together with Oliver Kirsch) and finalized the manuscript. TB, $\mathrm{PM}$, and $\mathrm{PK}$ assisted with the design, production and testing of the technical components. FK, TB, PD, and PM carried out the field sampling. All co-authors (FK, TB, PM, PK, NB, PD, and WE) contributed to the study design and writing of the manuscript.

\section{FUNDING}

Internal funding was used in this project. NB acknowledges support from the SNF for ICOS-CH (grants 148992 and 173691).

\section{REFERENCES}

Anderson, K., and Gaston, K. J. (2013). Lightweight unmanned aerial vehicles will revolutionize spatial ecology. Front. Ecol. Environ. 11, 138-146. doi: 10.1890/ 120150

Barker, M. G., and Pinard, M. A. (2001). Forest canopy research: sampling problems, and some solutions. Plant Ecol. 153, 23-38. doi: 10.1023/ a:1017584130692

Brockerhoff, E. G., Barbaro, L., Castagneyrol, B., Forrester, D. I., Gardiner, B., González-Olabarria, J. R., et al. (2017). Forest biodiversity, ecosystem functioning and the provision of ecosystem services. Biodiver. Conserv. 26, 3005-3035. doi: 10.1007/s10531-017-1453-2

Chapin, F. S. III., Matson, P. A., and Mooney, H. A. (2002). Principles of Terrestrial Ecosystem Ecology (New York, NY: Springer).

Congalton, R. G., and Green, K. (2009). Assessing the Accuracy of Remotely Sensed Data: Principles and Practices, 2nd Edn. Boca Raton, FL: CRC Press.

Curran, P. J. (1989). Remote sensing of foliar chemistry. Remote Sens. Environ. 30, 271-278. doi: 10.1016/0034-4257(89)90069-2

Hallé, F., Cleyet-Marrel, D., and Ebersolt, G. (2000). Le Radeau des Cimes: l'exploration des canopées Forestières. Paris: JC Lattès.

Huete, A., Miura, T., Yoshioka, H., Ratana, P., and Broich, M. (2014). Indices of Vegetation Activity. in Biophysical Applications of Satellite Remote Sensing, ed J. M. Hanes (Berlin; Heidelberg: Springer), 1-41. doi: 10.1007/978-3-642-250477_1

Jensen, J. R. (2007). Remote Sensing of the Environment: An Earth Resource Perspective, 2 Edn. Upper Saddle River, NJ: Pearson Prentice Hall.

Kokaly, R. F., Asner, G. P., Ollinger, S. V., Martin, M. E., and Wessman, C. A. (2009). Characterizing canopy biochemistry from imaging spectroscopy and its application to ecosystem studies. Remote Sens. Environ. 113, S78-S91. doi: 10. 1016/j.rse.2008.10.018

Larcher, W. (2003). Physiological Plant Ecology: Ecophysiology and Stress Physiology of Functional Groups, 4 Edn. Berlin: Springer.

Lenth, R. (2018). Estimated Marginal Means, aka Least-Squares Means. Available online at: https://cran.r-project.org/web/packages/emmeans/index.html

Lowman, M. D., and Moffett, M. (1993). The ecology of tropical rain forest canopies. Trends Ecol. Evol. 8, 104-107

\section{ACKNOWLEDGMENTS}

The authors are deeply indebted to Oliver Kirsch who, as a first year undergraduate student at ETH Zurich, designed the first prototype from which the present system was developed. Oliver Kirsch died in a helicopter accident at age 25 as a flight instructor in Honolulu, Hawaii, on 16 October 2017. Thus, we devote this paper to his enthusiasm for flying that initiated this project. We thank Joseph P. McFadden, University of California at Santa Barbara, for comments on an earlier version of the manuscript.

\section{SUPPLEMENTARY MATERIAL}

The Supplementary Material for this article can be found online at: https://www.frontiersin.org/articles/10.3389/ffgc.2018. 00002/full\#supplementary-material

Video S1 | A video (2:27 min, Supplementary Material 1) is linked to this article demonstrating the operation of the FTTS in the field. Additional technical information, including mechanical and electronical drawings, which will be made available subject to a CC-BY-SA Creative Commons attribution license 4.0, can be obtained via e-mail to FITS@usys.ethz.ch (TB and PM).
Muul, I., and Liat, L. B. (1970). Vertical zonation in a tropical rain forest in Malaysia: method of study. Science 169, 788-789. doi: 10.1126/science.169. 3947.788

Nakamura, A., Kitching, R. L., Cao, M., Creedy, T. J., Fayle, T. M., Freiberg, M., et al. (2017). Forests and their canopies: Achievements and horizons in canopy science. Trends Ecol. Evol. 32, 438-451. doi: 10.1016/j.tree.2017.02.020

Niinemets, U. (2012). Optimization of foliage photosynthetic capacity in tree canopies: towards identifying missing constraints. Tree Physiol. 32, 505-509. doi: 10.1093/treephys/tps045

Parker, G. G., Smith, A. P., and Hogan, K. P. (1992). Access to the upper forest canopy with a large tower crane. BioScience 42, 664-670. doi: 10.2307/1312172

R Core Team (2016). R: A Language and Environment for Statistical Computing. Vienna: R Foundation for Statistical Computing.

Searle, S. R., Speed, F. M., and Milliken, G. A. (1980). Population marginal means in the linear model: an alternative to least squares means. Am. Statist. 34, 216-221. doi: 10.1080/00031305.1980.10483031

Stork, N. E., Wright, S. J., and Mulkey, S. S. (1997). Craning for a better view: the canopy crane network. Trends Ecol. Evol. 12, 418-420. doi: 10.1016/s01695347(97)01205-6

Sutton, S. L. (2001). Alice grows up: canopy science in transition from Wonderland to Reality. Plant Ecol. 153, 13-21. doi: 10.1023/a:1017574 411128

Sweney, W. J., and Jones, A. E. (1974). Methods for sampling foliage and insect populations of the beech forest canopy. N.Z. J. Forest. Sci. 5, 119-122.

Conflict of Interest Statement: The authors declare that the research was conducted in the absence of any commercial or financial relationships that could be construed as a potential conflict of interest.

Copyright (C) 2018 Käslin, Baur, Meier, Koller, Buchmann, D’Odorico and Eugster. This is an open-access article distributed under the terms of the Creative Commons Attribution License (CC BY). The use, distribution or reproduction in other forums is permitted, provided the original author(s) and the copyright owner(s) are credited and that the original publication in this journal is cited, in accordance with accepted academic practice. No use, distribution or reproduction is permitted which does not comply with these terms. 